This is the supplementary material associated with the article

\title{
Refining Fuel Composition of RP-3 Chemical Surrogate Models by Reactive Molecular dynamics and Machine Learning
}

Table S1. Fuel composition of the derived surrogate models used in this work [1-3].

\begin{tabular}{|c|c|c|c|c|c|c|c|c|c|}
\hline \multirow{3}{*}{$\begin{array}{c}\text { Number of } \\
\text { derived } \\
\text { surrogate } \\
\text { models }\end{array}$} & \multicolumn{2}{|c|}{ 2-component model [1] } & \multicolumn{3}{|c|}{ 3-component model [2] } & \multicolumn{4}{|c|}{ 4-component model [3] } \\
\hline & \multicolumn{2}{|c|}{$\begin{array}{c}\text { n-decane } \\
\text { 1,2,4-trimethylbenzene }\end{array}$} & \multicolumn{3}{|c|}{$\begin{array}{c}\mathrm{n} \text {-dodecane } \\
\text { 1,3,5-methylcyclohexane } \\
\text { propylbenzene }\end{array}$} & \multicolumn{4}{|c|}{$\begin{array}{c}\text { n-decane } \\
\text { n-dodecane } \\
\text { ethylcyclohexane } \\
\text { p-xylene }\end{array}$} \\
\hline & 89 & 11 & 66 & 18 & 16 & 40 & 42 & 13 & 5 \\
\hline 1 & 30 & 70 & 27 & 30 & 43 & 93 & 2 & 1 & 4 \\
\hline 2 & 53 & 47 & 95 & 1 & 4 & 85 & 11 & 1 & 3 \\
\hline 3 & 72 & 28 & 67 & 22 & 11 & 12 & 23 & 15 & 50 \\
\hline 4 & 53 & 47 & 62 & 22 & 16 & 61 & 15 & 12 & 12 \\
\hline 5 & 77 & 23 & 51 & 15 & 34 & 95 & 2 & 1 & 2 \\
\hline 6 & 89 & 11 & 43 & 44 & 13 & 81 & 11 & 2 & 6 \\
\hline 7 & 36 & 64 & 21 & 61 & 18 & 61 & 33 & 1 & 5 \\
\hline 8 & 89 & 11 & 24 & 25 & 51 & 8 & 75 & 14 & 3 \\
\hline 9 & 95 & 5 & 5 & 57 & 38 & 40 & 17 & 32 & 11 \\
\hline 10 & 94 & 6 & 15 & 33 & 52 & 55 & 4 & 38 & 3 \\
\hline 11 & 51 & 49 & 84 & 3 & 13 & 97 & 1 & 1 & 1 \\
\hline 12 & 46 & 54 & 26 & 60 & 14 & 20 & 6 & 65 & 9 \\
\hline 13 & 76 & 24 & 87 & 12 & 1 & 60 & 5 & 5 & 30 \\
\hline 14 & 85 & 15 & 93 & 2 & 5 & 15 & 46 & 16 & 23 \\
\hline 15 & 18 & 82 & 13 & 53 & 34 & 10 & 26 & 11 & 53 \\
\hline 16 & 99 & 1 & 77 & 7 & 16 & 44 & 27 & 14 & 15 \\
\hline 17 & 32 & 68 & 76 & 16 & 8 & 56 & 33 & 4 & 7 \\
\hline 18 & 4 & 96 & 58 & 5 & 37 & 91 & 1 & 1 & 7 \\
\hline 19 & 84 & 16 & 9 & 81 & 10 & 14 & 7 & 4 & 75 \\
\hline 20 & 91 & 9 & 73 & 3 & 24 & 81 & 15 & 2 & 2 \\
\hline 21 & 98 & 2 & 13 & 31 & 56 & 61 & 14 & 3 & 22 \\
\hline 22 & 80 & 20 & 96 & 3 & 1 & 17 & 68 & 13 & 2 \\
\hline 23 & 72 & 28 & 76 & 21 & 3 & 17 & 46 & 3 & 34 \\
\hline 24 & 59 & 41 & 41 & 8 & 51 & 58 & 15 & 11 & 16 \\
\hline 25 & 97 & 3 & 33 & 4 & 63 & 40 & 8 & 13 & 39 \\
\hline 26 & 59 & 41 & 86 & 6 & 8 & 67 & 26 & 1 & 6 \\
\hline 27 & 92 & 8 & 11 & 22 & 67 & 72 & 10 & 13 & 5 \\
\hline 28 & 39 & 61 & 77 & 17 & 6 & 12 & 53 & 34 & 1 \\
\hline 29 & 91 & 9 & 36 & 9 & 55 & 5 & 70 & 1 & 24 \\
\hline 30 & 77 & 23 & 27 & 66 & 7 & 23 & 17 & 15 & 45 \\
\hline
\end{tabular}


Table S2. Error analysis for ignition delay time at equivalence ratio of 1 and different pressure of 1, 2, $4 \mathrm{~atm}$ for the refined surrogate and its parent 3-component surrogate of RP-3 fuel.

\begin{tabular}{ccccc}
\hline \multirow{2}{*}{$\begin{array}{c}\text { Pressure } \\
(\mathrm{atm})\end{array}$} & $\begin{array}{c}\text { Mean Absolute Error (MAE) of } \tau_{\text {ign }}(\mu \mathbf{s}) \\
\text { between surrogate calculation and real } \\
\text { RP-3 fuel experiments }\end{array}$ & $\begin{array}{c}\text { Mean Absolute Error (MAE) of } \log \tau_{\text {ign }}(\mu \mathbf{s}) \\
\text { between surrogate calculation and real RP-3 } \\
\text { fuel experiments }\end{array}$ \\
\cline { 2 - 5 } & $\begin{array}{c}\text { optimal } \\
\text { surrogate model }\end{array}$ & $\begin{array}{c}\text { its parent } \\
\text { surrogate }\end{array}$ & $\begin{array}{c}\text { optimal } \\
\text { surrogate model }\end{array}$ & $\begin{array}{c}\text { its parent } \\
\text { surrogate }\end{array}$ \\
\hline $1 \mathrm{~atm}$ & 83.0549 & 158.8333 & 0.0616 & 0.0859 \\
$2 \mathrm{~atm}$ & 36.7519 & 75.2649 & 0.0463 & 0.1132 \\
$4 \mathrm{~atm}$ & 153.5405 & 213.9880 & 0.0674 & 0.1185 \\
\hline
\end{tabular}

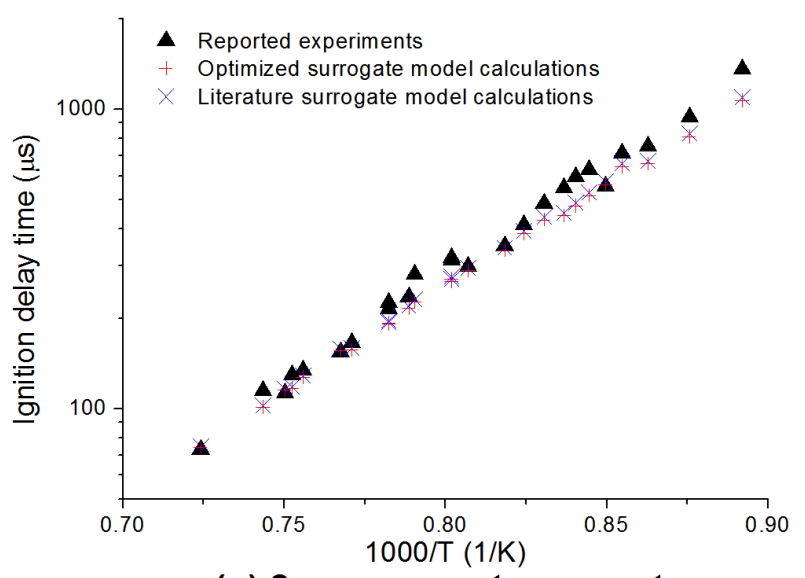

(a) 2-component surrogate

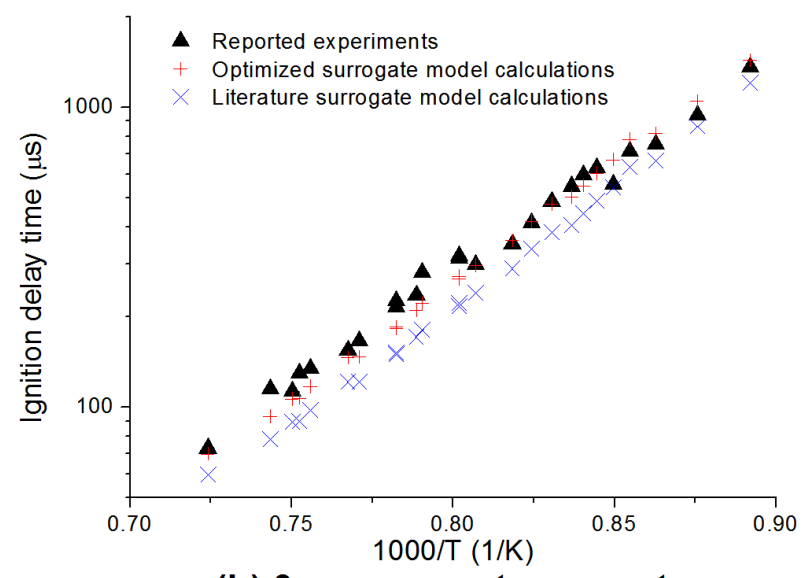

(b) 3-component surrogate

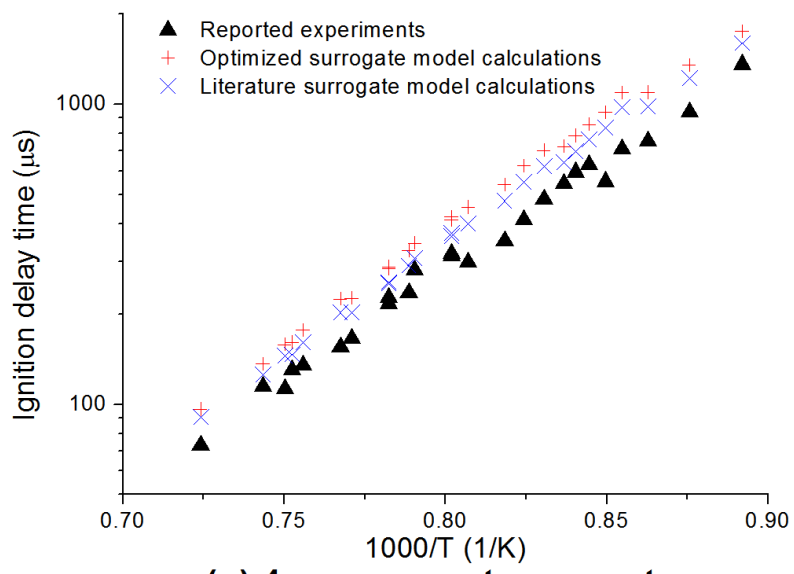

(c) 4-component surrogate

Figure S1. Comparison of ignition delay time at equivalence ratio of 1 between the experimental data of real RP-3 fuel and that of the optimal RP-3 surrogate model with composition predicted by LightGBM for all the three surrogates and their parent RP-3 surrogate model calculated by CHEMKIN. 


\section{References}

(1) Zhang, C.; Li, B.; Rao, F.; Li, P.; Li, X. A shock tube study of the autoignition characteristics of RP-3 jet fuel. Proc. Combust. Inst. 2015, 35, 3151-3158.

(2) Xu, J.; Guo, J.; Liu, A.; Wang, J.; Tan, N.; Li, X. Construction of Autoignition Mechanisms for the Combustion of RP-3 Surrogate Fuel and Kinetics Simulation. Acta Phys. -Chim. Sin. $2015,31,643-652$.

(3) Zheng, D.; Yu, W.; Zhong, B. RP-3 Aviation Kerosene Surrogate Fuel and the Chemical Reaction Kinetic Model. Acta Phys. -Chim. Sin. 2015, 31, 636-642. 